\title{
Safer prescribing in intensive care: designing a system to reduce errors
}

\author{
Kathryn Went \\ University of Dundee \\ Queen Mother Building \\ Dundee DD1 4HN \\ +44 (0) 1382388084
}

kwent@computina.dundee.ac.uk

\begin{abstract}
Prescribing in intensive care is a complex process involving a number of disciplines working in a highly stressful clinical environment. Within the National Health Service this process is generally written down manually. Errors are made each year as a consequence of illegible or incorrect prescriptions.
\end{abstract}

This research investigates engaging users from multi-disciplines in the design process to result in a system that is usable and demonstrates a reduction in prescribing errors

\section{Keywords}

User-centred design, evaluation, user interfaces, prototyping

\section{INTRODUCTION}

The National Programme for IT in the NHS, which is expected to exceed £12bn [1], has been widely criticized for running behind schedule and producing solutions that have not always been deemed as satisfactory. One of the reasons for this alleged failure is not engaging with the clinicians during the initial stages of the design [1].

This research looks at involving users throughout the development of an electronic prescribing system to result in a successful end product which is not only usable but reduces the errors made in prescribing in intensive care.

\section{BACKGROUND}

Approximately 200 million prescriptions are generated in NHS hospitals each year in the UK [3]. The majority of these prescriptions are handwritten.

The Department of Health estimates that one in 10 patients admitted to NHS hospitals will be unintentionally harmed, with medication errors being the second highest reported cause of harm. [2]

A medication error is defined as a mistake, slip or lapse made when medicines are prescribed, dispensed or used.

It is currently estimated that between $44,000-98,000$ deaths per year are attributable to medication errors in America [4] and an unknown number in the UK [2] which is likely to be

(c) K. Went

2007 Published by the British Computer Society

Volume 2 Proceedings of the 21st BCS HCI Group

Conference HCI 2007, 3-7 September 2007, Lancaster

University, UK

Devina Ramduny-Ellis \& Dorothy Rachovides (Editors) comparable.

Medication errors are believed to be a significant problem in the intensive care unit. Patients are frequently prescribed a large number of drugs, often in complicated combinations. This complexity increases the risk of medication errors, which may have catastrophic consequences. [3]

It is therefore imperative that in such critical environments there is an appropriate prescribing system in place to ensure accuracy and provide a clear audit trail.

\section{PROCESS}

The Intensive Care Unit (ICU) at Ninewells Hospital Dundee approached the University of Dundee to request the investigation of replacing the paper based prescribing system used in the ICU ward with an electronic version.

\subsection{Identifying the errors made}

Initially a survey, to identify the prescribing errors that occur in the ICU, was carried out. This allowed an understanding to be formed of how these errors could be prevented.

The survey took place over a 15 week period, 21 November 2005 until 7 March 2006. In this time 90 prescription charts were viewed and surveyed for 68 different patients. The survey consisted of checking the chart against 15 standards and documenting whether these standards were met or not. The standards were derived from the local hospital Safe and Secure Handling of Medicines Policy [5].

All of the charts viewed contained at least one non-compliance. Of the 1,921 individual medical prescriptions viewed 30\% contained at least one deviation, with some prescriptions containing several.

\subsection{Involving the users}

It is commonly accepted that involving users in the development process results in a successful end product.

To ensure that the software created catered for the needs of the clinicians, nurses and pharmacists a clinical development team was established consisting of a consultant in anesthesia and intensive care, a consultant in anesthesia, an intensive care fellow, the principal clinical pharmacist for critical care, the intensive care specialist liaison nurse and the practice education facilitator.

To encourage and then later maintain engagement and buy-in by the clinical staff, monthly meetings were held with the team to discuss and evaluate the development of the prototype. These meetings consisted of the members of the clinical development team and a group from the University of Dundee and were led 
by the author. Meeting agendas were circulated in advance of meetings providing an opportunity for items to be added. The agendas were well received by the team as they allowed the meetings to be structured and thus were very productive.

\subsection{System design}

The aim of the design was to produce an error-free interface that captured the users' requirements and produced a positive user experience.

The system design employed an iterative approach, using rapid prototyping, responding to feedback from the regular meetings of the multi disciplinary team, to inform changes and additions to the design.

After observing staff in ICU the preliminary design was created using paper prototypes. Once evaluated this design was implemented as a working prototype. Rapid prototyping was employed to ensure that usable prototypes were produced whilst maintaining the engagement of the users. Many design iterations were completed as a result of the feedback given from the 30 evaluation sessions held.

Discussions during these sessions often resulted in the identification of new requirements, which may not have been discovered if this approach was not taken. Due to the number of new requirements identified it was suggested by a member of the team to create a wish list of requirements that could be developed at a future stage. This was suggested as the system was being developed as part of a $\mathrm{PhD}$ research project and whilst it was important to implement requirements that would make a direct impact on patient safety not all would necessarily reduce errors.

\subsection{Evaluation}

Evaluation sessions held with the clinical development team consisted of a cognitive walkthrough of the software followed by a feedback session chaired by the author.

To gain feedback from users' outwith the group, several drop-in sessions were held. To accommodate with the ICU staffs busy workload, these sessions took no longer than half an hour comprising of a walkthrough of the software followed by a series of open-ended and closed questions.

A member of staff from the School of Computing performed 3monthly heuristic evaluations on the design to allow any usability issues to be highlighted.

An evaluation was carried out, with 22 prescribers outwith the team, which compared errors made with the paper system against the electronic system. Initial results suggested that the electronic system not only reduced non compliances made in the prescription process but was favoured by the users, who considered it "very user friendly and intuitive". Figure 1 shows the output of the evaluation for one of the subjects on both the electronic and paper system.

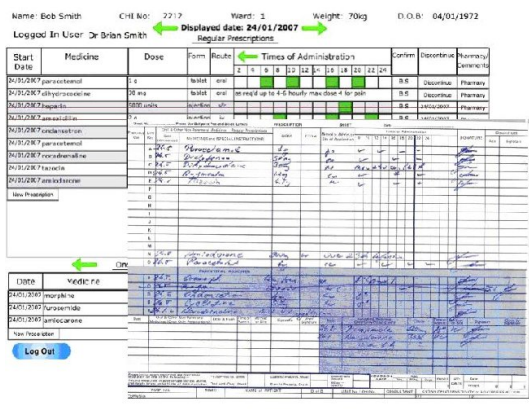

Figure 1. Electronic and paper system

Engaging the users of the system during the design process resulted in a working prototype which users considered "very clear and familiar" and "a lot better" than the current procedure. Adopting this iterative and responsive approach to the design of the evolving prototype resulted in the users developing a strong sense of ownership for the developed prototype.

\section{SUMMARY}

By applying usability techniques and involving the end users in the design it is hoped to show that the solution produced reduces errors made in Intensive Care prescribing in addition to creating a piece of software which the users accept and enjoy using.

The main contribution to the HCI field that this study is making is confirming that genuine buy-in and involvement is crucial to the development of a new error-free system accepted by the users.

\section{FUTURE PLANS}

Preliminary findings show that there is a reduction in errors with the electronic system but this will need to be demonstrated when used in the real life setting. The feedback received about the electronic system has been very positive but once again will need to be evaluated when used with actual patients in Intensive Care.

\section{ACKNOWLEDGMENTS}

My PhD supervisors, Prof Peter Gregor and Prof Ian Ricketts. The team of users at Ninewells Hospital, Dundee: Patricia Antoniewicz, Deborah A Corner, Dr. Judith Joss, Ann Mathewson, Dr. Shaun McLeod, Dr. Alfred J Shearer

\section{REFERENCES}

[1] Cross, M. Benefits of $£ 12$ bn IT programme in NHS are "unclear," MP s say. BMJ 2007;334(7598):815

[2] Department of Health. A safer place for patients: Learning to improve patient safety. London: The Stationery Office, 2005.

[3] Department of Health. Building a safer NHS for patients: Improving Medication Safety. London: The Stationery Office, 2004.

[4] Kohn, L., Corrigan, J., Donaldson M. (1999). To Err is Human: building a safer health system. Washington, D.C.: National Academy Press, 1999

[5] Royal Pharmaceutical Society. The safe and secure handling of medicines: a team approach. London: RPSGB, 2005. 